\title{
Research on fault diagnosis method of distribution transformer based
}

\section{on MFCC and HMM}

\author{
Hao Qin ${ }^{1, a}$, Wenyou Zhou², Minzhi Zhang², Pengxiang Liu² \\ ${ }^{1}$ Foshan Power Supply Bureau of Guangdong Power Grid Company, Foshan Guangdong \\ 2Shunde Power Supply Bureau of Guangdong Power Grid Company, Foshan Guangdong
}

aemail: 278115095@qq.com

\begin{abstract}
Keywords: distribution transformer; Mel coefficient of frequency (MFCC); hidden Markov model (HMM) ; fault diagnosis; acoustic signal

Abstract: The acoustic signal of the distribution transformer contains a much of information about the state of the transformer operation. Therefore, the fault diagnosis method of distribution transformer based on MFCC and HMM is proposed. The acoustic diagnosis process of transformer fault can be divided into three parts: (1) pre-acquisition and pre-processing of signal, (2) the MFCC and its first-order difference coefficient of the transformer acoustical signal are extracted as the characteristic quantities of the acoustic signals of distribution transformers, (3) the hidden Markov model (HMM) is used to classify and identify the acoustic signals. This method is verified by experiments and the influence of several common environmental disturbance on fault diagnosis is considered. The results show that the method can be applied to the fault diagnosis of the distribution transformer, and it has a certain anti-interference ability.
\end{abstract}

\section{Introduction}

Distribution transformer is one of the important equipment in the power system ${ }^{[1]}$, with large number and the number is large, scattered location and heavy inspection task, thus there's an urgent need for a convenient and accurate fault diagnosis method. It can be seen that different fault of transformer causes different sound according to DL/T 573-2010 "Power Transformer Overhaul Guidelines" for common abnormal condition checks, so the transformer fault can be diagnosed by sound.

The use of acoustic signals for fault diagnosis is convenient, non-contact, low cost, and has been applied in the gears, bearings and other equipment fault diagnosis ${ }^{[2-4]}$. However, there are few researches on transformer fault diagnosis method based on acoustic signal. The feature extraction of transformer acoustic signal is concentrated on time-frequency characteristic ${ }^{[5,6]}$, and the effect is very limited. Therefore, Mel coefficient of frequency (MFCC) and its first-order difference coefficient are adopted in this paper, which is based on simulated human ear acoustics so as to characterize the characteristics of transformer acoustic signals better ${ }^{[7,8]}$.

After the fault feature is extracted, whether the fault type can be identified is also the key problem of transformer fault diagnosis. Hidden Markov Model (HMM) ${ }^{[9]}$ is a statistical model of time series with strong classification ability, which can deal with the acoustic signal contains statistical information and timing information better and is widely used in speech recognition [10,11]. 
In view of this, the transformer fault diagnosis is realized by extracting MFCC and its first-order differential coefficient of acoustic signal as the characteristic parameters, identifying and classifying the transformer acoustic signals based on the hidden Markov model.

\section{Feature Extraction of Transformer Acoustic Signals Based on MFCC}

\section{Audio signal preprocessing}

For a transformer acoustic signal $\mathrm{x}(\mathrm{n})$, the preprocessing is as follows.

Normalization processing, that is, the transformer acoustic signal amplitude is limited to [-1, 1], to address size differences among the different samples.

Pre-emphasis, the normalized signal $\mathrm{x}_{1}(\mathrm{n})$ is passed through a high-pass filter. The pre-emphasis signal $\mathrm{x}_{2}(\mathrm{n})$ is:

$$
x_{2}(n)=x_{1}(n)-a \times x_{1}(n-1)
$$

Where, $\mathrm{a}=0.97$.

(1)Overlapping the sub-frame, that is to overlap the sub-frame with the long segment of the audio signal. The acoustic signal length of each frame is approximately $30-40 \mathrm{~ms}$. And in order to prevent the large adjacent signal change, there will be two adjacent acoustic signal overlap between the region, about $1 / 3$ the length of each frame of acoustic signal.

\section{Extract of Mel coefficient of frequency and its first order difference coefficient}

The frequency domain signal $\mathrm{Y}(\mathrm{k})$ is obtained by performing a discrete Fourier transform (DFT) on each frame of the pre-processed audio signal $y(n)$ :

$$
Y(k)=\sum_{n=0}^{N-1} y(n) e^{-j 2 \pi n k / N} \quad 0 \leq k \leq N
$$

Where, $\mathrm{N}$ is the number of sampling points.

The energy spectrum of $\mathrm{Y}(\mathrm{k})$, that is, $\mathrm{Y}(\mathrm{k})$ is first squared, and then filtered through the triangular bandpass filter accoding to the average frequency distribution of Mel frequency. The transfer function of the mth bandpass filter can be expressed as:

$$
H_{m}(k)=\left\{\begin{array}{cc}
0 \quad k<f(m-1) \text { or } k>f(m-1) \\
\frac{2(k-f(m-1))}{(f(m+1)-f(m-1))(f(m)-f(m-1))}(f(m-1) \leq k \leq f(m)) \\
\frac{2(f(m+1)-k)}{(f(m+1)-f(m-1))(f(m+1)-f(m))}(f(m) \leq k \leq f(m+1))
\end{array}\right.
$$

Where, $\sum_{m=0}^{M-1} H_{m}(k)=1, f(\mathrm{~m})$ is the center frequency of the triangular filter which satisfies:

$$
\operatorname{Mel}(f(m+1))-\operatorname{Mel}(f(m))=\operatorname{Mel}(f(m))-\operatorname{Mel}(f(m-1))
$$

The relationship between the physical frequency $f$ and the $\operatorname{Mel}$ frequency $\operatorname{Mel}(f)$ is as follows:

$$
\operatorname{Mel}(f)=2595 \times \lg \left(1+\frac{f}{700}\right)
$$

The logarithmic energy of each filter output is calculated: 


$$
E(m)=\ln \left(\sum_{k=0}^{N-1} H_{m}(k) \times|Y(k)|^{2}\right) \quad 0 \leq m<M
$$

Mel coefficient of frequency can be calculated after the discrete cosine transform (DCT):

$$
C(n)=\sum_{m=0}^{M-1} E(m) \cos \left(\frac{\pi n(m-0.5)}{M}\right) \quad 0 \leq n \leq M
$$

Where $n$ is the number of MFCC, $C(n)$ is the nth MFCC coefficient, $E(m)$ is the log power spectrum of the transformer acoustical signal, $M$ is the number of triangular filters and $M=24$ here. What's more, take $n=12$ to calculate MFCC coefficient $C(n)$. Then MFCC coefficients are first differentiated according to Eq.7, and a set of MFCC difference coefficients $D(n)$ is calculated:

$$
D(n)=\sum_{i=-k}^{k} i C(n+i) / \sum_{i=-k}^{k} i^{2}
$$

Where, $\mathrm{k}=2$.

\section{Classification and Recognition of Transformer Acoustic Signal Based on HMM}

For an HMM model of transformer acoustic signal, it can be simplified as a 3-element array:

$$
\lambda=\{\pi, A, B\}
$$

Where: $\pi$ represents the initial probability distribution vector, $A$ represents the state transition probability matrix, $B$ represents the observation probability matrix.

The process of classification and recognition of transformer acoustic signals based on HMM is the process of training and identifying HMM of transformer acoustic signals. In this paper, HMM is trained by Baum-Welch algorithm, which is a common HMM algorithm. Viterbi algorithm is used for its identification. The flow chart is shown in Fig.1:

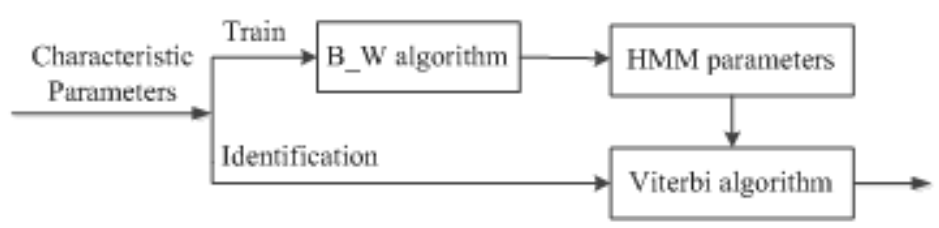

Fig.1. The principle diagram of the HMM model

HMM recognition algorithm of transformers acoustic signals, that is the Baum-Welch algorithm, which can be described as: A set of transformer acoustic signal characteristic quantities $Q=Q 1$, $\mathrm{Q} 2, \ldots, \mathrm{Qt}$ and an HMM initial value $\lambda=(\pi, A, B)$ is given to make sure that the probability $\mathrm{P}(\mathrm{Q} \mid \lambda)$ of the transformer characteristic value $\mathrm{Q}$ with respect to $\lambda$ is maximized. In order to obtain the optimal solution, the iterative operation is needed until $\mathrm{P}(\mathrm{O} \mid \lambda)$ converges, that is, the HMM model parameters no longer change.

After the model of each transformer acoustic signal is trained, the Viterbi algorithm is used to identify the acoustic signal to be measured, and the probability of the characteristic of the acoustic signal passing through each transformer acoustic signal HMM model can be calculated, and the model with the largest output probability is taken as the recognition result. However, false results may be produced because the environment interference is relatively large, thus the threshold of the 
calculated probability need to be set. It is only within the threshold that the acoustic signal is determined to be the acoustic signal..

\section{Acoustic diagnostic process of transformer fault}

The acoustic diagnosis process of transformer fault can be divided into three parts: (1) pre-acquisition and pre-processing of signal, (2) extract of MFCC and its first-order difference coefficient of transformer acoustic signal, (3) MFCC and its first-order difference coefficients are input to the HMM for model training as feature vector, and the HMM classifier is used to identify the fault. The acoustic diagnosis process of transformer fault is shown in Fig. 2 .

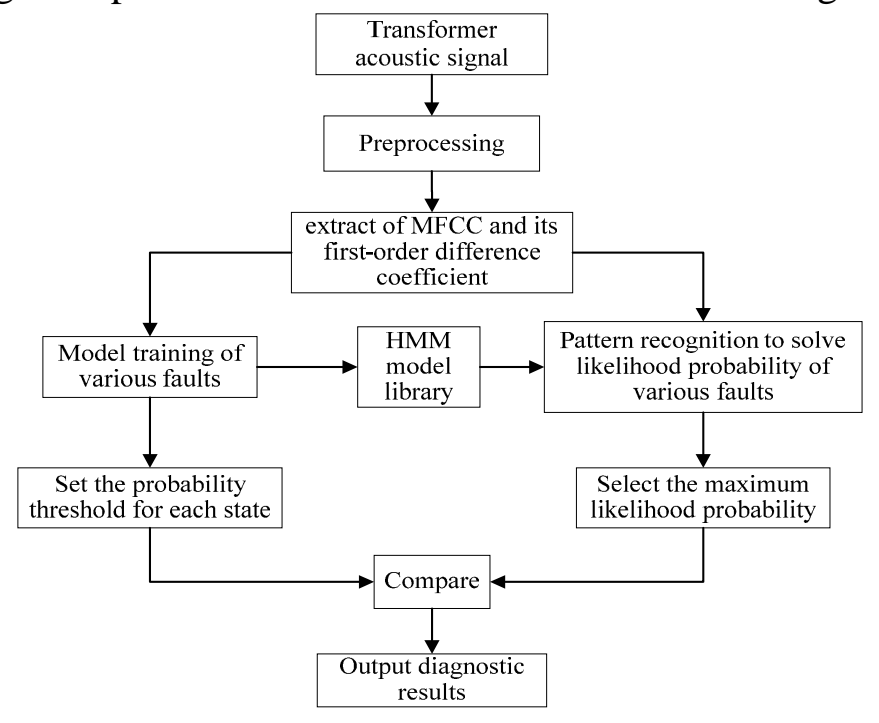

Fig.2. Flow chart of acoustic detection diagnosis of transformer faults

The likelihood probability threshold values $\mathrm{m} 1, \mathrm{~m} 2 \ldots$ are set for the training models for each fault. The signal to be tested is put into the pattern recognition to solve likelihood probability of a variety of fault, the state with the maximum likelihood probability is selected as the probable fault state. And then compares the obtained likelihood probability with the likelihood threshold corresponding to the fault, and if it is smaller than the threshold value, then it is judged as the fault. Otherwise it is judged as interference signal.

\section{Experimental Verification}

In order to verify the effectiveness of the proposed method, the normal sound and the loose core sound of the $10 \mathrm{kV}$ oil-immersed transformer was collected. According to the method mentioned in [12], the internal discharge test of the transformer was carried out, and the sound of the discharge of the needle plate in the transformer oil was collected. The acoustic signal is collected by the acoustic sensor, and the sampling rate is $24 \mathrm{k}$. Each acoustic signal was collected 10 times, each time $1 \mathrm{~min}$. Using matlab software for data signal processing.

The MFCC and the first-order difference coefficients are extracted for the collected acoustic signals according to the above-mentioned method. The order of the Mel filter is 24 , and s frame consists of 1024 sampling points, about $40 \mathrm{~ms}$ for a frame. The normal acoustic signal of transformer is shown in Fig.3, the characteristics of transformer loosening acoustic signal is shown in Fig.4, the characteristics of the transformer discharge acoustic signal is shown in Fig.5. It can be seen that the characteristics of the normal acoustic signal and the loosening acoustic signal are consistent with each frame, and the acoustic signal of the transformer discharge has certain randomness due to its discharge, and its acoustic signal characteristics have certain differences. 


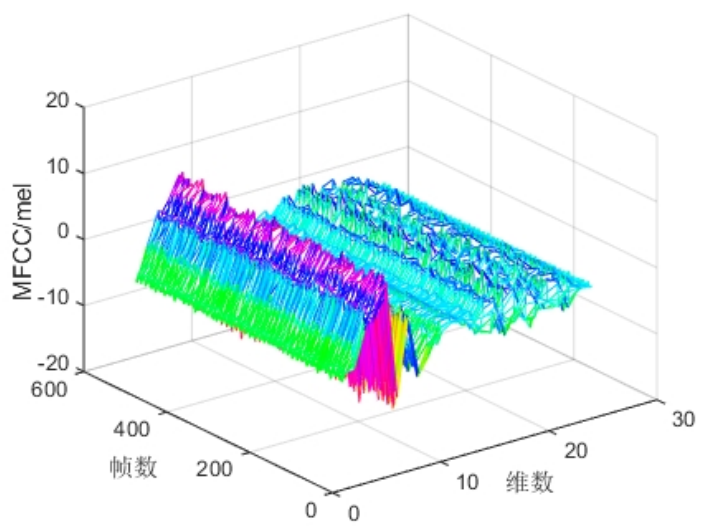

Fig.3. MFCC feature of transformer normal acoustic signal

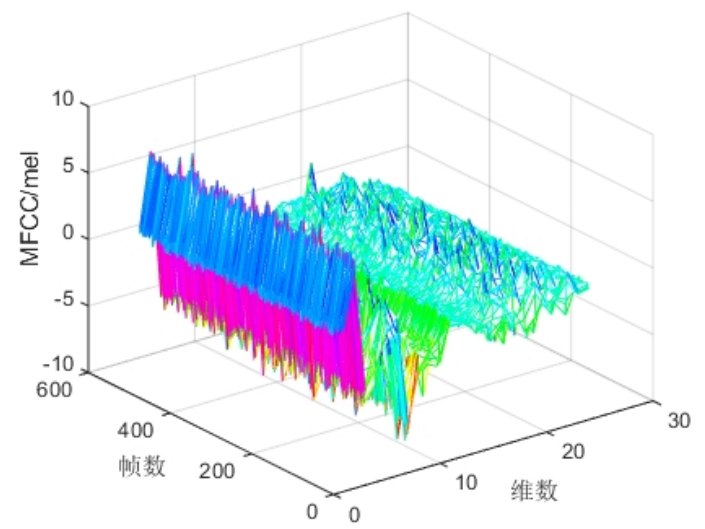

Fig.4. MFCC feature of transformer loose acoustic signal

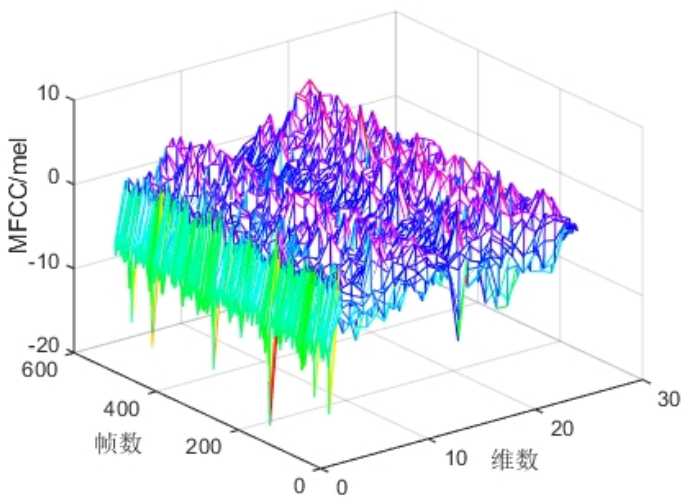

Fig.5. MFCC feature of transformer discharge acoustic signal

3000 frames from each acoustic signal sample were chosen as training samples for HMM training. Note that the selected samples are selected from 10 samples of data, respectively. After training and recognition by HMM, the sample threshold of each sound is set, where the probability threshold of the normal acoustic signal is -105 , the probability threshold of the loosening acoustic signal is -100 , and the probability threshold of the discharging acoustic signal is -110 .

As the transformer in which the environment is more complex, there are some interference with acoustic signals. In order to verify the anti-interference ability of the method proposed in this paper, a car horn sound, rain, people speaking are collected. Each audio 1min, feature extraction is done the same for these acoustic signals, for convenience, the three acoustic signals are referred as environmental interference signal. 
The fault acoustic signal of the transformer is identified by HMM, and 800 frames of the test samples are selected. Among them, the normal acoustic signal of the transformer is 200 frames, the loosening signal of the transformer is 200 frames, the discharging signal is 200 frames and the environmental interference signal is 200 frames. Part of the recognition results are shown in Table 1. In Table 1, M1 is the output probability of the normal acoustic signal, M2 is the output probability of the loose signal, M3 is the output probability of the discharge acoustic signal, Inf is infinity. The final recognition rate is shown in Table 2.

Table 1. Part identification results of transformer acoustic signal

\begin{tabular}{|c|c|c|c|c|}
\hline \multirow{2}{*}{$\begin{array}{c}\text { Type of test } \\
\text { sample }\end{array}$} & \multicolumn{3}{|c|}{ Output probability through } & \multirow{2}{*}{$\begin{array}{c}\text { Determine the } \\
\text { target type }\end{array}$} \\
\hline & M1 & M2 & M3 & \\
\hline $\begin{array}{l}\text { Normal acoustic } \\
\text { signal (1) }\end{array}$ & -94.7144 & -168.5125 & -210.5591 & Normal \\
\hline $\begin{array}{l}\text { Normal acoustic } \\
\text { signal (2) }\end{array}$ & -95.0567 & -170.5893 & -189.2178 & Normal \\
\hline $\begin{array}{l}\text { Normal acoustic } \\
\text { signal (3) }\end{array}$ & -101.6287 & -191.5874 & -182.8831 & Normal \\
\hline $\begin{array}{c}\text { Loosening acoustic } \\
\text { signal (1) }\end{array}$ & -177.9991 & -85.0325 & -162.8956 & Loose \\
\hline $\begin{array}{c}\text { Loosening acoustic } \\
\text { signal (2) }\end{array}$ & -178.1077 & -82.5667 & -162.4417 & Loose \\
\hline $\begin{array}{c}\text { Loosening acoustic } \\
\text { signal (3) }\end{array}$ & -163.0116 & -80.9624 & -169.4064 & Loose \\
\hline $\begin{array}{c}\text { Discharge acoustic } \\
\text { signal (1) }\end{array}$ & -164.7231 & -311.7992 & -106.9070 & Discharge \\
\hline $\begin{array}{c}\text { Discharge acoustic } \\
\text { signal (2) }\end{array}$ & -176.1853 & -261.7842 & -89.2321 & Discharge \\
\hline $\begin{array}{c}\text { Discharge acoustic } \\
\text { signal (3) }\end{array}$ & -168.2739 & -333.9107 & -98.390 & Discharge \\
\hline $\begin{array}{c}\text { Interference } \\
\text { acoustic signal (1) }\end{array}$ & -16897.2512 & $-\operatorname{Inf}$ & $-\operatorname{Inf}$ & Rejection \\
\hline $\begin{array}{c}\text { Interference } \\
\text { acoustic signal (2) }\end{array}$ & -120.8547 & -302.1254 & -17862.2821 & Rejection \\
\hline $\begin{array}{c}\text { Interference } \\
\text { acoustic signal (3) }\end{array}$ & $-\operatorname{Inf}$ & -Inf & $-\operatorname{Inf}$ & Rejection \\
\hline
\end{tabular}


Table 2. Recognition rate of transformer fault acoustic signal

\begin{tabular}{|c|c|c|c|c|}
\hline Type & $\begin{array}{c}\text { Normal acoustic } \\
\text { signal }\end{array}$ & $\begin{array}{c}\text { Loosening } \\
\text { acoustic signal }\end{array}$ & $\begin{array}{c}\text { Discharge } \\
\text { acoustic signal }\end{array}$ & $\begin{array}{c}\text { Interference } \\
\text { acoustic signal }\end{array}$ \\
\hline $\begin{array}{c}\text { Recognition } \\
\text { rate }\end{array}$ & $92.5 \%$ & $87.5 \%$ & $83.5 \%$ & $95.5 \%$ \\
\hline
\end{tabular}

\section{Conclusion}

It can be seen from the experimental analysis that the fault acoustic diagnosis method of transformer based on MFCC and HMM proposed in this paper, which take the advantages of MFCC in simulating the characteristics of human ear acoustics and the advantages of HMM in processing the statistical information and timing information in acoustic signals, can realize better fault diagnosis of the transformer, and has strong resistance to environmental interference.

\section{References}

[1] Cao Dunkui, Xu Weizong, Ruan Guofang. Analysis and processing of transformer operation maintenance and fault[M]. Beijing: China Electric Power Press,2008.

[2] Zhang Ying, Su Xianzhang, Liu Zhansheng. Fusion method for processing non-contact multi-sensor acoustic signals in rolling bearing fault diagnosis[J].Journal of Vibrations and Shock,2012,16:188-192.

[3] Anami B S, Pagi V B, Acoustic signal-based approach for fault detection in motorcycles using chaincode of the pseudospectrum and dynamic time warping classifier[J]. IET Intelligent Transport Systems, 2014,8(1):21-27.

[4] Zhang Shangbin, Zhang Haibin, Ouyang Kesai, et al. Doppler distortion removal based on time-frequency amplitudematching method for fault diagnosis of train bearings acoustic signals[J]. Journal of Vibrations and Shock, 2016,14:101-106.

[5] Pan Liangliang, Zhao Shutao, Li Baoshu. Electrical equipment fault diagnosis based on acoustic wave signal analysis[J]. Electric Power Automation Equipment, 2009,08:87-90.

[6] Pan Qingqing, Zhao Shutao, Pan Liangliang. Transformer Fault Diagnosis and Trend Prediction Based on Acoustic Processing[J] Transformer, 2009,02:62-66.

[7] Hossan M A, Memon S, Gregory M A. A novel approach for MFCC feature extraction[C]. Proc of the 4th International Conference on Signal Processing and Communication Systems. New York: IEEE Press, 2010: 1-5.

[8] Lǜ Xiaoyun, Wang Hong-xia. Abnormal audio recognition lgorithm based on MFCC and short-term energy[J]. Journal of Computer Applications,2010,03:796-798.

[9] Huang X D, Jack M A .Semi-continuous hidden Markov models for speech signals[J].

Computer Speech and Language, 1989, 3(2):239 -251.

[10] Dai Weiguo, Zhang Baohua, Cheng Yusheng. Ship-radiated noise recognition based on CHMM[J]. Technical Acoustics,2008,02:187-191. 
[11] Liu Hui,Yang Junan,Xu Xuezhong. A Novel Low Altitude Passive Acoustic Target Identify Approach Research Based on MFCC and HMM[J]. Journal of Projectiles, Rockets, Missiles and Guidance,2007,05:217-219+222.

[12] Dan Wengang, Chen Xiang-xun, Zheng Jian-chao. Measurment and analysis of pulse current of partial discharge in oil[J]. Power System Technology, 2000(06):37-40. 


\title{
Application and Outlook of In-situ Combustion for Developing Heavy Oil Reservoir
}

\author{
ZHANG Xishun ${ }^{1,}$, , LIN Chunqing ${ }^{2}$, GU Liming ${ }^{3}$, XIA Yinghu $^{4}$, GUO Zhaoxia ${ }^{5}$, \\ SHI Junfeng ${ }^{1}$, ZHAO Ruidong ${ }^{1}$, ZHANG Xin ${ }^{1}$, and CHEN Shiwen ${ }^{1}$ \\ ${ }^{1}$ Research Institute of Petroleum Exploration \& Development, CNPC, China \\ ${ }^{2}$ Research Institute of Petroleum Exploration \& Development, CNPC, China \\ ${ }^{3}$ Oil Development Department of Jidong Oilfield, CNPC, China \\ ${ }^{4}$ Supervision Station of Downhole Operation Department of Shanshan Oilfield, CNPC, China \\ ${ }^{5}$ Bidding Center of Huabei Oilfield, CNPC, China \\ ashunzi-2003@163.com
}

Keywords: In-situ combustion, Heavy oil reservoir, Burning front, Ignition technique

Abstract. As the most potential development method of heavy oil reservoir, in-situ combustion has the maximal recovery factor due to its multiple displacement mechanisms. The paper presents the enhancing oil recovery mechanism of in-situ combustion through analyzing the combustion principle of crude oil in reservoir and displacement mechanism of burning front. Then the five key technologies of in-situ combustion were discussed primarily here on the basis of oil burning characteristic in reservoir, including the selecting criterion of in-situ combustion for heavy oil reservoir, ignition technique, monitoring technique of burning, combustion control technique of burning front, physical and mathematical simulation of in-situ combustion. From the discussion, present technique status was presented, the key problems were exposed, and the technique demands and long term potential were brought forward. As In-situ combustion being in the ascendant, the paper summarized its enhancing oil recovery mechanism, the techniques demanding and the outlook in the future, which provided the most advanced technologies and point out the developing direction about it.

\section{Introduction}

Heavy oil in reservoir buried in several kilometers depth has high viscosity which makes it flow difficult and results in a low recovery factor. So enhancing oil recovery(EOR) of heavy oil reservoir was one of the most important topics researchers concerned Injecting heat medium to heat oil and reduce viscosity was the popular method to develop heavy oil reservoir, including hot water flooding and steam injection. The former results in water fingering and water flush inevitably due to the great mobility ratio between oil and water and the later results in steam overlapping because of the low density of steam, which both result in a low recovery factor in developing heavy oil reservoir. Still, heat medium injection needs huge energy consumption ${ }^{[1]}$.

As a new EOR method, in-situ combustion was proposed and made great progress from the 30th last century. A fire lighter is needed and putted into the down hole of wellbore; air is injected continually for some day until the heated air firing the oil layer around wellbore ${ }^{[1-4]}$. Then air is injected according to the injection project, which makes the burst front driven ahead from injection well to production well, and then heated oil, distilled oil and cracked oil will flow into production well naturally. From theoretical analysis, the burst front could generate multiple displacement effects. No water fingering and steam overlapping would be brought since no heat medium injected, which results in higher recovery factor and shorten heat waste. Crude oil would be burned in in-situ combustion unavoidably, but the losses are mainly the components of bitumen and coke. In recent years, with some key techniques being broken through and heavy oil development being in the ascendant resulted from energy shortage, in-situ combustion applied to develop heavy oil reservoir rapidly in many countries. 


\section{Mechanism of in-situ combustion}

As the heavy components of crude oil, Bitumen and coke was left and being the fuel through distilled and thermal cracked by burning front. The oxygen in air injected continually and regularly maintains the burning front moving from injection well to production well. As the main component, distilled and cracked light oil was driven ahead. Steam and $\mathrm{CO}_{2}$ were given birth to through the burning between oxygen with the fuel, which can generate the two conventional EOR effects of steam driving and $\mathrm{CO}_{2}$ miscible flooding respectively. From injection well to production well, the reservoir can be divided into five zones which as burning zone, coke zone, steam zone, light oil zone and originality zone sequentially as the fig1. So, in-situ combustion have multiple effects of displacements such as steam driving, hydrocarbon miscible flooding and $\mathrm{CO}_{2}$ miscible flooding, which result in a higher recovery factor than other EOR method ${ }^{[3-6]}$.

Temperature distribution
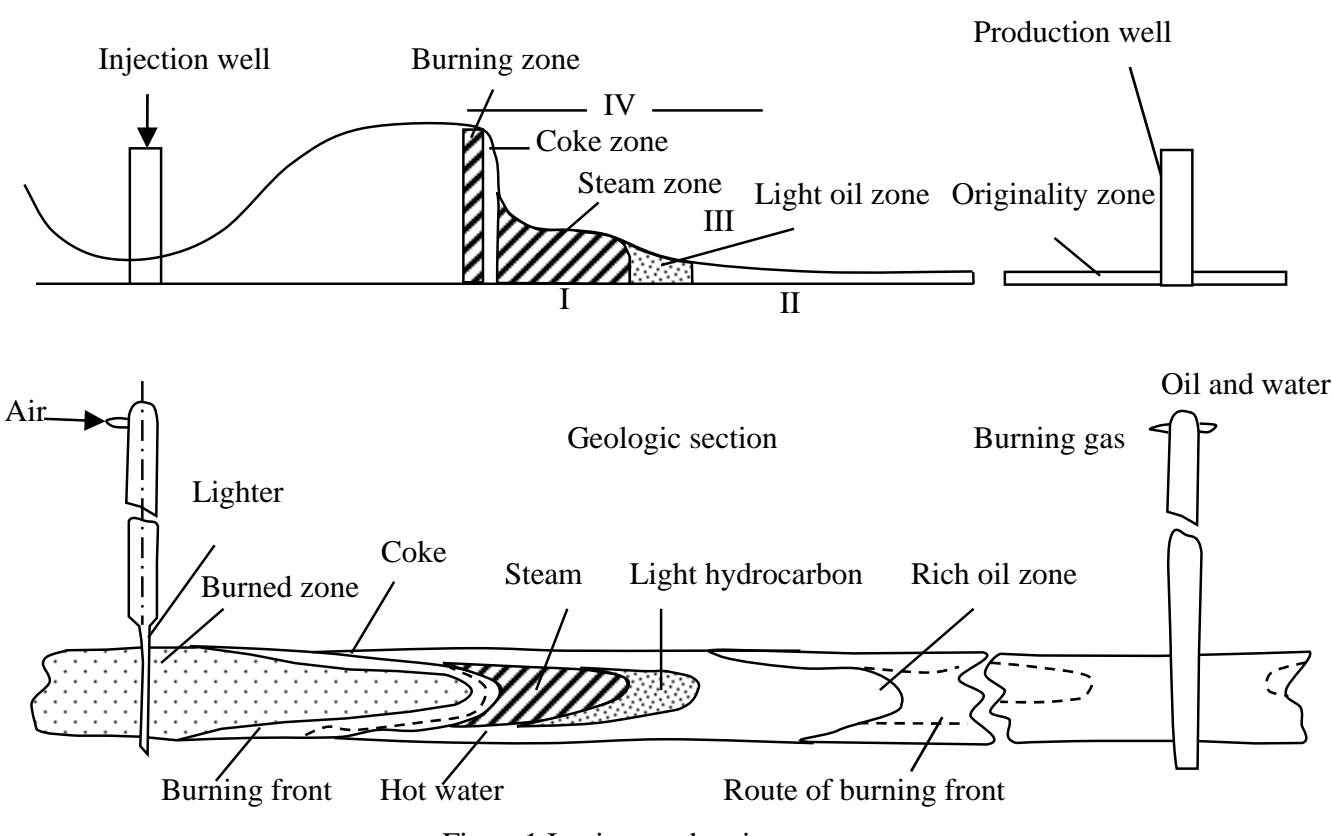

Figure1 In-situ combustion process

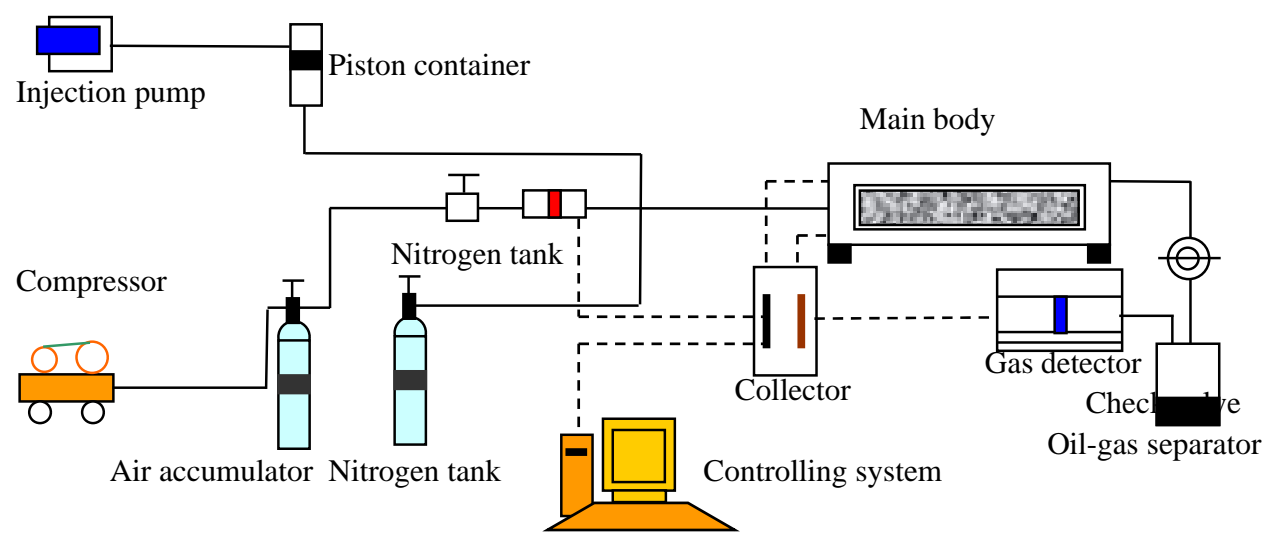

Figure2. The flow chart of 3D physical simulation

For the evolution behavior of crude oil in burning, thermo-gravimetry can be used to study the function mechanism. Different group and component in crude oil has different burning behavior, in which saturated hydrocarbon and aromatic hydrocarbon are in the extreme.

In general, the process of burning can be fallen into two phases: distillation phase and oxidation phase. The former generated in the temperature ranged $25 \sim 300{ }^{\circ} \mathrm{C}$, the later can be divided into three 
phases which as low temperature oxidation(LTO), middle temperature oxidation(MTO) and high temperature oxidation $(\mathrm{HTO})^{[6,7]}$.

As the lightest component, saturated hydrocarbon would burn firstly, which occurs in the phase of LTO and the loss be about in $89 \%$. The high components such as bitumen and coke lose very little in the phase. In the phase of MTO, the effect of saturated hydrocarbon decreased and the asphaltene and aromatic hydrocarbon began to bring into play. The chemical reactions are all gas phase reactions including the oxidation of cracked product, which produces the fuel of HTO. The product of MTO oxidized further and the HTO generated, in which bitumen and coke play the important roles and the loss accounts for $90 \%$. The reaction in HTO phase, a large amount heat was produced to burn coke, crack crude oil and heat saturated hydrocarbon, which were the EOR mechanism of in-situ combustion. In a word, saturated hydrocarbon has earlier and higher reactivity, which can be as the igniter and control the combustion front, bitumen and coke being the fuel, maintain the constancy of burning.

\section{Key techniques and its status}

From theoretical analysis to practical application, in-situ combustion must work out some key techniques including selecting criterion of candidate reservoir, firing technique, burning front monitoring and controlling, physical simulation and mathematical simulation.

Selecting criterion of candidate reservoir. Despite the face that in-situ combustion is one of the most scientific EOR methods, not all heavy oil reservoirs were suited to develop in it. As in-situ combustion being a high investment project, selecting criterion must be formulated before carry into execution, which bases on crude oil property, nature of rock and reservoir physical property. America Petroleum Institute (API), Gephen and Chuke etc. had presented some selecting criterions ${ }^{[4,5]}$ based on oil viscosity, reservoir thickness, reservoir depth, content of coke and so on. The values of these criterions are different for different researcher because they come from some successful example reservoirs of in-situ combustion. So, the values can not be applied generally.

D.V.Yannimaras introduced a selecting method ${ }^{[7]}$ through testing thermodynamics parameters of crude oil by accelerating rate calorimeter (ARC). ARC is the apparatus applied to study on reaction kinetics through adiabatic reaction, which can obtain the thermodynamics parameters of crude oil in a wide pressure range of 10.0 70.0MPa.

Arrhenius activation energy, index factor, reaction order, initial temperature and heat release degree can be tested by ARC on which we based to select the appropriate candidate reservoir to develop in in-situ combustion. Furthermore, if combustion tube testing combined to ARC, the selecting result would be more credible.

Ignition technique. Igniting the around reservoir and achieving stable burning is the first but the utmost step of in-situ combustion, which decides the effect of in-situ combustion. Ignition methods included self-ignited in reservoir and ignited manually. The former had few or no application because it's just appropriate to specially reservoir and the time of ignition is very long. For the later, electric heater ignition is the most general and advanced technique, in which clubbed form heater is popular. $\mathrm{Li}$ Youping, Li Shulan etc. invented SL-I high power electric heater ignition ${ }^{[8,9]}$. When ignition device was selected, corresponding ignition parameters must be decided reliably. Tetama and Haitama developed the calculated formula of igniting time through the energy balance of LTO. Li Shulan presented the predication model of air injecting rate, electrical parameters, and temperatures through simulating temperature and pressure distribution.

Monitoring and controlling techniques of burning front. After reservoir was ignited, monitoring and controlling burning was the significant task which maintains the burning front burning uniformly and driving firmly and results in a high recovery factor.

As the burning front driving ahead radically, different burning phase needs different air injection rate, which makes the burning stably and the sweep efficiency spread. Nelson and Mcniel developed a air injection model ${ }^{[9]}$, which has been accepted by researchers generally. The model divided the injection course into three stages: primary stage, steady stage and final stage. For the primary stage, the air injection rate can be calculated by linear function. When the injection rate increases to the 
maximum value, then injection keeps steadily in the value that is the steady stage. The steady stage maintains sited time, and then the injection rate can be calculated by linear descend function. The key parameters needed determined are the maximum value of injection rate and the time of steady stage kept.

Monitoring the burning front dynamically is the basis of analyzing and adjusting injection project. Dynamic monitoring of in-situ combustion can be classed into two parts. One part is production status monitoring of injection and production wells including pressures and temperatures of the both wells, injection rate, oil production rate, gas production rate etc. Furthermore, production cross-sectional testing should be considered through steady and unsteady state well testing. The monitoring parameters are same to water flooding wells', but should be obtained under high temperature condition. The other part is monitoring property and components of production liquid, especially the production gas components. Through the monitoring data, the burning condition can be analyzed and injection project can be adjusted in time.

Physical simulation technique of in-situ combustion. As the investment of in-situ combustion being huge, every in-situ combustion project needs laboratory physical simulation before execution. The physical simulation in laboratory includes combustion kettle testing, one dimension combustion tube testing and 3D combustion testing ${ }^{[10,11]}$.

Mathematical simulation of in-situ combustion. Mathematical model is needed to simulate the burning and production course of in-situ combustion since the mechanism is very complex and can be discovered by 3D combustion testing. Primitively, a three phases and five components (oxygen, nitrogen, $\mathrm{CO}_{2}$, water and oil) model had been developed and to simulate the flow of liquid and thermal transmission. With the advancing of mathematical theory and computer technology, CMG company developed a combustion simulator which consider many of components to simulate the flow law of liquid, thermal transmission, burning, gasification, cracking and cooling ${ }^{[12]}$. Furthermore, the displacement and production course can be simulated and predicted. For mathematical simulation, the testing data from physical simulation is very significant to the accuracy of predicted result.

\section{Technologies demand and long term potential}

From the analysis, all the key technologies above need improve if in-situ combustion wants to be a widespread development method for heavy oil reservoir ${ }^{[7-12]}$.

I For selecting criterion of candidate reservoir, at present the considered factors were few and empirical. More factors should be considered and comprehensive assessment method such as fuzzy mathematics should be applied to develop a scientific selecting criterion.

I For firing technique, ignition device appropriated for deep well, high temperature well and high water ratio well should be developed.

I As the high temperature and poly component in-situ combustion being, monitoring technique should break through the limitation of high temperature.

I With the development of 3D physical simulation, more influencing factors should be considered to make the simulation more analogous to practical combustion, such as physical similarity, burning front monitoring etc.

\section{Conclusions}

The paper presented the enhancing oil recovery mechanism of in-situ combustion through analyzing the combustion principle of oil in reservoir and displacement mechanism of burning front. Five zones were classed as burning zone, coke zone, steam zone, light oil zone and originality zone sequentially.

Five key technologies of in-situ combustion were discussed significantly here on the basis of heavy oil combustion principle in reservoir, including the selecting method of in-situ combustion reservoir, firing technique, monitor technique of burning front, combustion control technique, physical and mathematical simulation of in-situ combustion. 
Technologies demand and long term potential were pointed out based on the analysis of key techniques.

\section{References}

[1] Guan Wenlong, Wu Shuhong, Liang Jinzhong, et al. The research on engineering risk in combustion assisted gravity drainage based on indoor experiment. Journal of Southwest Petroleum University. 2009, 31(4), 67-72.

[2] Adegbesan K O, et a1. Low Temperature Oxidation Kinetic Parameters for In-Situ Combustion: Numerical Simulation. SPE 12004.

[3] Binder G G, et a1. Scaled Model Tests of In Situ Combustion in Massive Unconsolidated Sands : World Pet. Cong. Maxico City, 1967: 477-485

[4] Zhao Dongwei, Jiang Haiyan, Zhang qi. Dry combustion physical modeling of in-situ combustion. Oi1Drilling \& Production Technology, 2005, 27(1): 36-39.

[4] GRABOWSKI Janusz W, VINSOME Paul K, LIN Ran C, et al. A fully implicit general finite-difference thermal model for in-situ combustion and stearn. SPE8396, 1979.

[5] LIN C Y, CHEN W H, CULHAM W E. New kinetic thermal cracking of crude oil in in-situ combustion processes. SPE 13074, 1987.

[6] ONYEKENWOMO, PANDE K, RAMEY H J, et a1. Experimental and simulation studies of laboratory in-situ combustion recovery. SPE 15090, 1996.

[7] DU Dianfa, YAO Jun. Effect of development parameters on in-situ cornbustion after iniecting steam in Le an oil Field. Journd of the University of Petroleum, China, 2003, 27(2): 47-50.

[8] Kumar M. Simulation of laboratory in-situ combustion data and effect of process variation. SPE 16027, 1987.

[9] Crookstion R B, Culham W E. The numerical simulation model for thermal recovery process. SPEJ, 1979.

[10]Penberthy Jr W L, Ramey. Design and operation of laboratory combustion tubes. SPE 1290, 1966.

[11]Coats R, Lorimer S, Ivory J. Experimental and numerical simulations of a novel top down in-situ combustion process. SPE 30295, 1995.

[12] Jia Zhenyuan et al. Formation and distribution of hydrocarbon in carbonate. Beijing: Petroleum Industry Press, 1 989: 45-63. 